\title{
Double Minute Chromosomes Associated with Philadelphia Chromosome in Meyloid Leukemia. A Case Study
}

\section{Abolfazl Movafagh ${ }^{1 *}$, Mostafa Rezaei Tavirani ${ }^{2}$ and Mohamad Hasan Heidari ${ }^{3}$}

${ }^{1}$ Department of Medical Genetics, Faculty of Medicine, Shahid Beheshti University of Medical Sciences, Tehran, Iran

${ }^{2}$ Faculty of Paramedical Sciences, Shahid Beheshti University of Medical Sciences, Tehran, Iran

${ }^{3}$ Department of Medical Anatomy, Faculty of Medicine, Shahid Beheshti University of Medical Sciences, Tehran, Iran

\begin{abstract}
The Philadelphia chromosome was the first consistent cytogenetic abnormality observed in a human cancer Several characteristic chromosome rearrangements like double minute chromosomes are known to be associated with different subtypes of myeloid leukemia. Double Minute Chromosomes are the cytogenetic hallmarks of genomic rearrangements in cancers. Two cases, one with AML-M1 and the other with CML- blast crisis, associated with double minute chromosomes and Philadelphia chromosome is presented. The additional chromosome changes to primary chromosome abnormality may be influenced by other factor like chemotherapy. The identification of two new cases with co-expression of double minute chromosomes and $\mathrm{Ph}$ chromosome presented here for the first time in Iran together with large Mitelman database and other pertinent website reports is discussed.
\end{abstract}

Keywords: Co-expression; Ph; DM; Leukemia; Incidence

\section{Introduction}

Double minute chromosomes (DMs) were first described in a direct preparation of cells from patient with untreated brochogenic carcinoma [1]. Sait et al. [2] reported for the first time that, DMs originating from chromosome 1. Double Minute Chromosomes are the cytogenetic hallmarks of genomic amplification in cancers [3]. Furthermore, DMs derived from the breakpoint region of translocation event [4]. Although DMs found in a variety of human tumor cells, their presence in hematologic malignancies is rare. Also their role in leukemogenesis is not clear but they have been reported to be associated with rapid progression and short survival time [5]. Double Minute Chromosomes are thought to be involved in tumorigenesis and in drug resistance [6]. They are small chromatin particles that represent a form of extra chromosomal gene amplification [7].

The Philadelphia $(\mathrm{Ph})$ chromosome, or $\mathrm{t}(9 ; 22)$ is the hallmark of Chronic Myelogenious Leukemia (CML) [8] and acute myeloid leukemia [5]. The Ph generally appears as a primary at the onset of the disease. However, a late -developing or DMs has also been observed in rare cases of CML [9] and AML [5].

Here we present two leukemia cases revealed double minute chromosomes associated with $\mathrm{Ph}$ chromosome.

\section{Case Report}

Two patients with CML and AML have seen between 1994-2010, from Shahid Beheshti University of Medical Sciences and various private clinics, Tehran, Iran.

\section{Case 1}

A 23 years old housewife, in previous good health, presented with weight loss, tiredness with a history of weakness with 3 months medical treatment on chemotherapy. Her peripheral blood hemoglobin was $6.2 \mathrm{~g} / \mathrm{dl}$ with a platelet count of $100 \times 10^{9} / 1$ (differential: $77 \%$ blast, $1 \%$ neutrophils, $22 \%$ lymphocytes). The marrow aspirate was cellular with reduction in all normal marrow elements and marked increased in Sudan Black-positive myeloblast. Peripheral blood and bone marrow sample was submitted for chromosome analysis after 3 months from her initial chemotherapy. G-banding analysis in patient one, AML-M1 showed that all of 20 metaphases cells revealed both numerical and structural abnormalities, including 46,XX,t $(9 ; 22)+$ dmin; 45,XX,t $(9 ; 22),-15$ (Figure 1A). Sixteen of the 20 cells (80\%) also had 3-19 DMs in each cells. Her past medical history was no history of prior toxic exposure.

\section{Case 2}

In patient two, with CML- blast crisis, 28 years old female was admitted to our hospital because of leukocytosis and anemia. On admission also splenomeghaly was found, WBC $15.1 \times 10^{4} / \mathrm{mm}^{3}$, hemoglobin was $7.2 \mathrm{~g} / \mathrm{dl}$, RBC $224 \times 10^{4} / \mathrm{mm}^{3}$ and bone marrow examination revealed hypercellularity with promyelocyte $14.2 \%$ and myeloblast $49.7 \%$. She was presented at the time of cytogenetic study
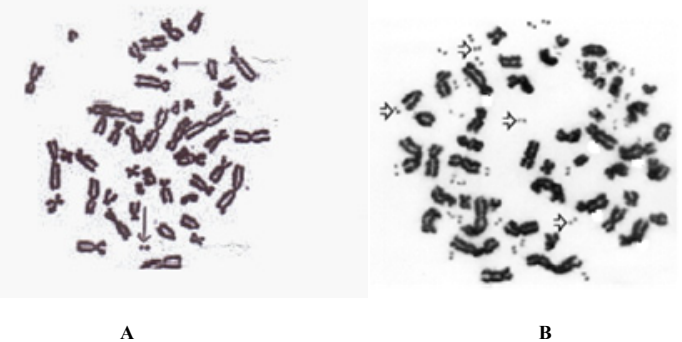

Figure 1: Giemsa staining bone marrow cell containing dmin in the metaphase of chronic and acute myeloid leukemia patients.

*Corresponding author: Abolfazl Movafagh, Ph.D, Associate Professor Department of Medical Genetics, Faculty of Medicine, Shahid Behesht University of Medical Sciences, Tehran, Iran, Fax +98(21) $2240067+98-21$ 23872572; E-mail: Movafagh_a@yahoo.com

Received March 24, 2012; Accepted May 28, 2012; Published May 30, 2012

Citation: Movafagh A, Tavirani MR, Heidari MH (2012) Double Minute Chromosomes Associated with Philadelphia Chromosome in Meyloid Leukemia. A Case Study. J Cancer Sci Ther 4: 144-145. doi:10.4172/1948-5956.1000129

Copyright: (C) 2012 Movafagh A, et al. This is an open-access article distributed under the terms of the Creative Commons Attribution License, which permits unrestricted use, distribution, and reproduction in any medium, provided the original author and source are credited. 
with intensive chemotherapy for 4 months. The patient smoked cigarettes for about 7 years. G-banding analysis revealed that 14 of the 20 metaphases cells $(70 \%)$ had $\mathrm{Ph}$ chromosome $+\mathrm{dmin}$ (Figure 1B). Her past medical history was notable only for occupation for cultivation as Farm Yard Manure. Fluorescence in situ Hybridization (FISH) analysis was not performed for both cases. Because of the lack of our patients response and / or shorter survival, did not allow us to identify whether $\mathrm{Ph}$ chromosome was a primary or a secondary event.

\section{Discussion}

As part of the large population base study have been published previously on CML and AML from own findings [10-13], the presence of DMs and $\mathrm{Ph}$ chromosome together, with two leukemia patients were identified in our laboratory was also observed in other parts of the world (http://cgapanci.nih.gov/chromosomes/Mitelman) [5]. These two new cases of DMs chromosome presented here is discussed in the following report [12], and utilizing the largest chromosome aberrations database in cancers (http://cgapanci.nih.gov/chromosomes/Mitelman). Also, Thomas et al. [5] documented 33 cases with DMs in acute myeloid leukemia in the current literature.

Chronic myelogenous leukemia is characterized by the Philadelphia chromosome which originated by reciprocal translocation $t(9 ; 22)$ [8]. The $\mathrm{Ph}$ chromosome generally appears as a primary changes at the onset of the disease. However, a late - developing or additional chromosome abnormality has been observed in rare cases of CML [9], AML [5,14] .

The relationship of DMs and malignancies seems well established and indeed DMs have not, so far, been observed in non malignant cell [3]. Double minute chromosomes have been found in various solid tumors, such as Colon, Pancreatic, Breast carcinoma, Brain tumors, and Neuroblastoma (http://cgapanci.nih.gov/chromosomes/ Mitelman). However, it appears to be less common in AML [5].

Past medical history of both cases presented here, recorded without history of any micro organism contaminations, but with the history of anemia. In a case 2, past medical history was notable only for occupation for cultivation as farm yard manure. It is coincident with previous reports indicating that, DMs do occur in leukemic patients without previous history of malignancy [15]. On the contrary, a relation between DMs and previous mutagenesis exposure has been suggested by some workers [16]. Thus for, suggest that the demonstration of DMs in patients with AML might be an indication that such patients have a previous history of malignant disease treated with irradiation or chemotherapy or both [16]. Expression of Ph chromosome may have been the first event in early myeloid stem cells in both of our patients, with the dmin occurring later due to the treatment with chemotherapy.

The identification of two new cases of DMs associated with $\mathrm{Ph}$ chromosome presented here are secondary. However, further studies and accumulation of new cases are needed in the hope of defining it as specific abnormalities in the field of leukemia.

\section{Acknowledgments}

I thanks, Dr. Fatemeh Isfahani, Dr. Abbas Hajfathali, Dr. Hamid Attarian, Dr. Morteza Ghadiani, Dr. Hamid Rezvani; Departments Medical Oncology, Internal Medicine. Thanks to Miss Niloofar Safavi for excellent technical assistance and material collection. This article is the part of thesis work.

Author has no conflict of interests.

\section{References}

1. Spriggs Al, Boddington MM, Clarke CM (1962) Chromosomes of human cancer cells. Br Med J 2: 1431-1435

2. Sait SN, Qadir MU, Conroy JM, Matsui S, Nowak NJ, et al. (2002) Double minute chromosomes in acute myeloid leukemia and myelodysplastic syndrome: identification of new amplification regions by fluorescence in situ hybridization and spectral karyotyping. Genes Chromosomes Cancer 34: 42-47.

3. Storlazzi CT, Lonoce A, Guastadisegni MC, Trombetta D, D'Addabbo P, et al. (2010) Gene amplification as double minutes or homogeneously staining regions in solid tumors: origin and structure. Genome Research 20: 1198-1206.

4. Reddy KS (2007) Double minutes (dmin) and homogeneously staining regions (hsr) in myeloid disorders: a new case suggesting that dmin form hsr in vivo. Cytogenet Genome Res 119: 53-59.

5. Thomas L, Stamberg J, Gojo I, Ning Y, Rapoport AP (2004) Double minute chromosomes in monoblastic (M5) and myeloblastic (M2) acute myeloid leukemia: two case reports and a review of literature. Am J Hematol 77: 55-61.

6. Von Hoff DD, McGill JR, Forseth BJ, Davidson KK, Bradley TP, et al. (1992) Elimination of extrachromosomally amplified MYC genes from human tumor cells reduces their tumorigenicity. Proc Natl Acad Sci U S A 89: 8165-8169.

7. Pappou EP, Ahuja N (2010) The role of oncogenes in gastrointestinal cancer Gastrointest Cancer Res (Suppl 1): S2-S15.

8. Zaccaria A, Testoni N, Valenti AM, Luatti S, Tonelli M, et al. (2010) Chromosome abnormalities additional to the Philadelphia chromosome at the diagnosis of chronic myelogenous leukemia: pathogenetic and prognostic implications. Cancer Genet Cytogenet 199: 76-80.

9. Morel F, Bris MJ, Herry A, Calvez GL, Marion V, et al. (2003) Double minutes containing amplified bcr-abl fusion gene in a case of chronic myeloid leukemia treated by imatinib. Eur J Haematol 70: 235-239.

10. Movafagh A, Varma N, Varma S (1996) Co-expression of two FAB-specific chromosome changes, $t(15 ; 17)$ and $t(8 ; 21)$, in a case of acute promyelocytic leukemia. Am J Hematol 72: 375-377.

11. Movafagh A, Hajifahthali A, Isfahani F, Attarian H, Ghadiani M, et al. (2009) Geographic heterogeneity of cytogenetic characteristics of acute myeloid leukemia: A comparative study of Iranian and Indian adult patients. IJCP 2: 85-89.

12. Movafagh A, Mirfakhraei R, Mousavi-Jarrahi A (2012) Frequent incidence of double minute chromosomes in cancers, with special up-to-date reference to leukemia. Asian Pac J Cancer Prev 12: 3453-3456.

13. Movafagh A, Hajifathali A, Zamani M (2011) Secondary Chromosomal Abnormalities of de novo Acute Meyloid Leukemia. A first report from the Middle East. Asian Pac J Cancer Prev 12: 2991-1994.

14. Fan Y, Mao R, Lv H, Xu J, Yan L, et al. (2011) Frequency of double minute chromosomes and combined cytogenetic abnormalities and their characteristics. J Appl Genet 52: 53-59.

15. Hartly SE, Toolis F (1980) Double minute chromosomes in a case of acute myeloblastic leukemia. Cancer Genet Cytogenet 2: 275-280.

16. Weh HJ, Zschaber R, Hossfeld DK (1982) Double minute chromosomes: a frequent marker in leukemic patients with a previous history of malignant disease? Cancer Genet Cytogenet 5: 279-280. 